\title{
TIR-1/SARM1 Inhibits Axon Regeneration
}

\author{
Victoria Julian ${ }^{1}$ and Alexandra B. Byrne ${ }^{1 *}$
}

${ }^{1}$ Department of Neurobiology, University of Massachusetts Medical School, Worcester, MA *Correspondence to: alexandra.byrne@umassmed.edu

Key words: TIR-1/SARM1, axon regeneration, axon degeneration, C. elegans

\begin{abstract}
$\underline{\text { Abstract }}$
An injured axon has two choices, regenerate or degenerate. In many neurons, the result is catastrophic axon degeneration and a failure to regenerate. To coerce the injured nervous system to regenerate, the molecular mechanisms that regulate both axon regeneration and degeneration need to be defined. We found that TIR-1/SARM1, a key regulator of axon degeneration, inhibits regeneration of injured motor axons. Loss of tir-1 function both reduces the frequency with which severed axon fragments degenerate and increases the frequency of axon regeneration. The increased regeneration in tir- 1 mutants is not a secondary consequence of its effects on degeneration. Rather, TIR-1 carries out each of these opposing functions cell autonomously by regulating independent downstream genetic pathways. While promoting axon degeneration with the DLK-1 mitogen activated protein kinase (MAPK) signaling cascade, TIR-1 inhibits axon regeneration by activating the NSY-1/ASK1 MAPK signaling cascade. Our finding that TIR-1 regulates both axon regeneration and degeneration provides critical insight into how axons coordinately regulate the two key responses to injury, consequently informing approaches to manipulate the balance between these responses towards repair.
\end{abstract}




\section{Introduction}

In injured neurons that are capable of repair, axons are broken into fragments with different fates. The axon segment that remains attached to the cell body regenerates and severed axon segments degenerate. However, this balance is disrupted in the central nervous system and in aged animals where axon regeneration is inhibited, resulting in a permanent loss of neuronal function $(1,2)$. Understanding the molecular mechanisms that coordinately regulate both axon regeneration and degeneration is critical to developing therapeutic approaches to repair the injured nervous system.

On the proximal side of the injury, functional axon regeneration requires the synchronized execution of multiple processes, including growth cone formation, axon guidance and synapse formation. Despite being recapitulations of well-characterized developmental processes, the precise molecular mechanisms that regulate axon regeneration remain incompletely understood. Those that have been identified are subdivided into two broad categories: intrinsic and extrinsic. Intrinsic regulators of transcription, transport, translation, and signaling are complemented and opposed by extrinsic regulators of myelin dependent inhibition and scar formation (3-8). Manipulating either its intrinsic or extrinsic environment is sufficient to modestly increase an injured axon's ability to regenerate $(7,9-11)$.

On the distal side of the injury, the most notable endogenous regulator of axon degeneration is Sterile Alpha and Toll/interleukin-1 resistance (TIR) Motif Containing 1 (SARM1/dSarm), which is essential for injury-induced axon degeneration of Drosophila and mouse axons (12, 13). SARM1/dSarm promotes axon degeneration cell-autonomously, by depleting $\mathrm{NAD}^{+}$and ATP, interacting with the BTB/BACK domain protein Axundead, and by interacting with mitogenactivated protein kinase (MAPK) signaling cascades, which in turn regulate calpain activation, SCG10/Stathmin 2 proteolysis, mitochondrial dysfunction, cytoskeletal disruption, and axon 
fragmentation (14-21). Whether the C. elegans SARM1/dSarm homolog, TIR-1, also regulates injury-induced axon degeneration is not known; however, in the absence of injury, SARM1, dSarm, and TIR-1 promote disease- and age-associated neurodegeneration (22-25). Despite the prominent role of SARM1/dSarm in the injury response, its function is currently understood to be restricted to the severed axon fragment distal to the injury site.

Here we report that not only does TIR-1/SARM1 promote injury-induced axon degeneration, it also inhibits axon regeneration. Specifically, in response to injury, TIR-1 functions with the NSY1/ASK1 MAPK signaling cascade and the terminal transcription factor DAF-19/RFX to inhibit regeneration of the proximal axon fragment. In parallel, TIR-1 functions independently from NSY1/ASK1 signaling and with the DLK-1/DLK MAPK pathway, to promote degeneration of the distal axon fragment. Together, our results indicate that axon regeneration and degeneration are both regulated by TIR-1, and that TIR-1 carries out these two opposite functions via two divergent, and therefore separable downstream pathways. Our findings reveal a mechanism for spatial and temporal control of MAPK signaling after axon injury, which can be manipulated to shift the injury response towards repair.

\section{$\underline{\text { Results }}$}

\section{TIR-1/SARM1 inhibits axon regeneration}

We asked whether TIR-1, the C. elegans homolog of SARM1/dSarm, regulates the injury response and found that when GABA motor axons of tir-1 loss of function and wild-type animals were severed at the midline, significantly more axons regenerated in the absence of tir-1 (Figure 1 BD). We tested two putative null alleles of $t i r-1$; the $q d 4$ allele is a 1,078 base pair deletion spanning 
exons 8, 9 and 10 and the $\operatorname{tm} 3036$ allele is a smaller 269 base pair deletion in exon 8 that results in an early stop codon $(26,27)$. Both the $q d 4$ and tm3036 deletions disrupt the C-terminal Toll/interleukin-1 Receptor (TIR) domain, which is essential for TIR-1 function (28) (Figure 1A). Therefore, TIR-1 inhibits regeneration of injured motor axons.

Successful regeneration of $C$. elegans motor axons includes growth cone formation, axon extension, and responsiveness to cues that guide the axon to the dorsal nerve cord. To determine which stage of the regenerative process is inhibited by TIR-1, we grouped individual injured axons into categories depending on whether it initiated regeneration and how far it regenerated towards the dorsal nerve cord (Figure $1 \mathrm{E}, \mathrm{F}$ ). We found a dramatic increase in both the number of axons that initiated regeneration and in the number of axons that regenerated the full distance to the dorsal nerve cord in tir-1 mutants compared to wild-type animals (Figure 1F). These results indicate TIR1 inhibits the initiation of axon regeneration and that the regenerating axons are able to successfully extend and reach the dorsal cord.

C. elegans have one other TIR domain containing protein, which is TOL-1(29). To determine whether regulation of axon regeneration is a general function of TIR domain containing proteins, we compared regeneration in the presence and absence of tol-1. We found no significant difference between the number of axons that regenerated in tol-1(nr2033) null mutants and wild-type animals (Supplementary Figure 1). Therefore, TIR-1 is a TIR-domain containing protein with a specific role in axon regeneration.

\section{Double injury induces axon degeneration of $C$. elegans GABA motor neurons}

The finding that TIR-1 inhibits axon regeneration is unexpected since SARM1 and dSarm promote the opposite response to injury, axon degeneration $(12,13)$. To determine if TIR-1 also regulates injury-induced degeneration in C. elegans, we needed to examine the consequences of mutating 
tir-1 in manipulations that induce degeneration. Although the above axotomy assay, in which an axon is severed once, is extremely useful for investigating the genetic and cellular mechanisms that regulate axon regeneration (30), it is not as robust a model of injury-induced axon degeneration. The obstacle is that while proximal segments, which remain attached to the cell body, regenerate in the 24 hours following injury (31), the distal segments, which are no longer attached to the cell body, remain largely intact (32). This prompted us to develop an assay with which both injury-induced motor axon degeneration and axon regeneration can be investigated simultaneously in vivo. Here, rather than severing axons once, we severed individual axons in two places, approximately $25 \mu \mathrm{M}$ apart, which creates a middle fragment that degenerates (Figure $2 \mathrm{~A}$ ). The degeneration has similar morphological and temporal features as Wallerian degeneration, where after a brief latent period, the middle axon segment beads, fragments, and is eventually cleared (Figure 2B). We tested how reliably double injury induces degeneration of the middle fragment by quantifying the percentage of severed axons whose middle fragment either degenerated (complete clearance), partially degenerated ( $>65 \%$ of middle fragment degenerated) or largely remained intact $(>80 \%$ of middle fragment remained). In wild-type axons, $90.2 \%$ of middle fragments degenerated 24 hours after double axotomy (Figure 2C). There was no significant difference between the number of middle fragments when visualized with either cytosolic or myristoylated and membrane bound GFP (Figure 2D). Together, this data suggests that the observed degeneration is an active process, not caused by a leaky cytosolic reporter and that the double injury reliably induces axon degeneration that can be investigated in vivo and with single-neuron precision. 


\section{TIR-1 promotes injury-induced and constitutive axon degeneration}

In the absence of tir-1, 68\% of middle fragments degenerated compared to $86 \%$ in wild-type animals (Figure 3A). In contrast, axons overexpressing tir- 1 were beaded and severely fragmented, even when not injured (Figure 3B,C,D). Upon closer examination, we found significantly less GFP expression along the axon commissures and dorsal nerve cord of tir- 1 overexpressing animals compared to non-transgenic controls (Figure 3E,F). Together, these data indicate TIR-1 promotes, but is not absolutely required for injury-induced axon degeneration. In addition, elevation of tir-1 expression is sufficient to induce severe and chronic motor axon degeneration, even in the absence of axotomy.

\section{TIR-1 inhibits axon regeneration independently from its role in axon degeneration}

Loss of tir-1 function enhanced regeneration of axons that had been severed twice compared to wild-type axons (Figure 3G). Therefore, TIR-1 inhibits axon regeneration after double injury, as it does after single injury. To determine whether the presence of the middle fragment influenced axon regeneration we grouped axons into one of two categories 24 hours after double injury: (1) completely degenerated middle fragment or (2) intact or only partially degenerated middle fragment. We found significantly fewer wild-type axons regenerated when the middle fragment degenerated compared to wild-type axons that did not (Figure $3 \mathrm{H})$. Together, these data indicate degeneration of the middle fragment inhibits axon regeneration. Of note, the number of axons that regenerated in tir- 1 mutants was similar between the injury models and regardless of whether the middle fragment had degenerated when injured twice (Figure 3H). Therefore, TIR-1 inhibits regeneration and degeneration of injured motor axons; however, its role in regeneration is not secondary to an inhibitory relationship between the two. 


\section{TIR-1 functions cell autonomously in GABA motor neurons to regulate both axon regeneration and axon degeneration}

We next asked how TIR-1 regulates axon regeneration and degeneration, and how it achieves specificity of function. TIR-1 is expressed and executes several functions in various C. elegans tissues including the nervous system, intestine, and epidermis $(28,29,33)$. To determine whether TIR-1 is expressed in GABA axons, we first CRISPR tagged tir- 1 with mCherry (Supplementary Figure 2A). With this single copy integration, we observed low level fluorescence throughout the intestine, epidermis, and sheath cells of the animal, but not in GABA motor neurons. (Supplementary Figure 2B). However, in a transgenic strain expressing multiple copies of tir$1:: m$ Cherry under its endogenous promoter, we also observed TIR-1::mCherry expression in the cytoplasm of GABA motor neurons (Supplementary Figure 2C). Together these data indicate TIR1 is expressed widely in tissues throughout the animal including in GABA motor neurons, where it is expressed at relatively low levels.

To determine whether TIR-1 achieves specificity of function by acting in different tissues to inhibit regeneration and promote degeneration, we asked whether TIR-1 functions in GABA axons to exclusively regulate one process or the other. We expressed tir- 1 cDNA specifically in GABA motor neurons or in the epidermis and found that GABA neuron specific expression rescued the enhanced regeneration observed in tir-1(qd4) mutants, while epidermis specific expression did not (Figure 3F). Similarly, GABA motor neuron specific expression of tir-1 cDNA rescued axon degeneration in tir-1(qd4) mutants, while epidermis specific expression of tir-1 did not (Figure 3A). Together, these data indicate TIR-1 functions cell autonomously in GABA neurons to both inhibit axon regeneration and promote axon degeneration after injury. 


\section{TIR-1 regulates axon regeneration with the NSY-1 - PMK-1 mitogen-activated protein kinase signaling cascade}

We next asked whether TIR-1 might direct multiple responses to injury by activating different downstream signaling events in each axon fragment. TIR-1 and SARM1/dSarm execute several cellular functions, including cleavage of $\mathrm{NAD}^{+}$, activation of MAPK signaling and the Axundead protein, as well as regulation of calcium signaling, gene transcription and autophagy $(14,15,24$, 25, 28). This informed a candidate approach to dissect the genetic pathways in which TIR-1 regulates both axon regeneration and degeneration. We found that loss of the MAP kinase kinase kinase $n s y-1 / A s k 1$ or its downstream MAP kinase $p m k-1 / p 38$ enhanced axon regeneration after double injury (Figure 4A,B). These regeneration phenotypes were not significantly enhanced by deletion of tir- 1 . Therefore, tir- 1 functions in the same genetic pathway as $n s y-1$ and $p m k-1$ to regulate axon regeneration after double injury (Figure 4A).

We also investigated whether TIR-1 regulates axon regeneration in coordination with the Dual leucine zipper MAP kinase kinase kinase DLK- 1, which is essential for axon regeneration (34, 35). Loss of tir- 1 function did not suppress the axon regeneration phenotype of either the $d l k-1$ null mutant or the downstream pmk-3 p38 MAPK null mutant, indicating tir-1 does not function downstream of $d l k-1$ signaling (Figure 4C). As with any gene that is essential for a given function, epistasis analysis with $d l k-1$ is complicated by the fact that it does not determine whether endogenous $d l k-1$ function is downstream or independent from another gene. However, in combination with the finding that loss of $n s y-1$ and $p m k-1$ result in the same amount of regeneration as loss of tir-1, our data indicate that the $n s y-1-p m k-1$ MAPK pathway does not act synthetically or in parallel to the $d l k-1-p m k-3$ MAPK pathway to mediate tir-1 function in axon regeneration. 
Together these results indicate that TIR-1 functions with the NSY-1 - PMK-1 MAPK pathway to regulate axon regeneration.

\section{TIR-1 inhibits axon regeneration by regulating the RFX-type transcription factor DAF-19}

During development, TIR-1 specifies the mature neuronal identity of AWC neurons downstream of the calcium/calmodulin dependent protein kinase II delta (CaMKII) ortholog, UNC-43. It does so by forming a postsynaptic protein complex with NSY-1 and UNC-43 $(28,36)$. We found that a null mutation in unc-43(e408) phenocopied the increased axon regeneration of tir-1(qd4) mutants and addition of the unc-43(e408) mutation to tir-1(qd4) mutants did not further enhance regeneration. Therefore $u n c-43$ and tir- 1 function in the same genetic pathway to inhibit axon regeneration (Figure 4D).

CaMKII has previously been found to regulate axon regeneration and developmental axon outgrowth, in part by regulating transcription of effector genes (37-39). However, the signaling pathways and set of transcription factors that mediate the effects of CaMKII activation on target gene expression during regeneration are not known. Given the genetic interactions between tir-1, unc-43, nsy-1, and pmk-1 we hypothesized that UNC-43 might regulate axon regeneration via TIR1 and NSY-1 dependent transcription. We examined this possibility by testing the regenerative ability of animals with predicted null mutations in the cAMP-dependent Transcription Factor atf$7 / A T F 2 / C R E B 5$ and in the Regulatory Factor $\mathrm{X}$ transcription factor $d a f-19 / R F X$, two transcription factors regulated by $n s y-1$ signaling $(24,27,40)$. Loss of atf-7 function did not affect GABA axon regeneration, consistent with previous studies of PLM mechanosensory axon regeneration (Supplementary Figure 3) (41). However, loss of daf-19 function increased regeneration to the same extent as loss of tir- 1 and did not enhance the regeneration phenotype of the tir- 1 null mutants 
(Figure 4D). Together, these data are consistent with a model in which an unc-43/tir-1/nsy-1/pmk1/daf-19 signaling cascade regulates axon regeneration.

\section{TIR-1 promotes axon degeneration via a separate MAPK signaling cascade}

To determine whether tir-1 also regulates axon degeneration with the nsy-1-pmk-1 MAPK pathway, we measured the presence or absence of degeneration 24 hours after double injury in the absence of the pmk-1 MAPK (Figure 5A). Loss of pmk-1 did not have a degeneration phenotype on its own and double tir-1; pmk-1 null mutants phenocopy the tir-1 single null mutant. This lack of genetic interaction indicates that tir- 1 functions independently from $p m k-1$ to promote axon degeneration after injury.

To determine which genes do function with tir-1 to promote axon degeneration, we returned to our candidate approach. DLK MAPK-signaling functions with SARM1/dSarm to regulate axon degeneration in mice and flies $(16,17,42-45)$. In our assay, loss of either $d l k-1$ or its downstream MAP kinase pmk-3 did not affect axon degeneration on their own, however they did rescue axon degeneration in tir-1 mutants (Figure 5B). This result suggests that the decreased axon degeneration phenotype of tir-1 mutants depends on DLK-1 pathway activity. However, due to the high amount of axon degeneration in wild-type animals it is possible that there is an upper threshold in our assay that prevents us from seeing enhanced degeneration above wild-type levels. Therefore, to determine whether $d l k-1$ does function with tir- 1 to regulate axon degeneration, we asked whether loss of $d l k-1$ suppresses the spontaneous axon degeneration phenotype of animals that overexpress tir-1 (Figure 5C,D). We observed an average of 16 GABA axons in wild-type animals and only 2 axons in wild-type animals that overexpressed TIR-1 (Figure 5C,D). Although $d l k-1$ mutants have a wild-type number of axons, loss of $d l k-1$ rescued the number of axons in the tir-1 overexpression background (Figure 5C,D). These data indicate $d l k-1$ is required for tir- 1 
dependent axon degeneration. Therefore, TIR-1 regulates axon regeneration and axon degeneration with distinct MAPK signaling cascades.

\section{$\underline{\text { Discussion }}$}

Understanding how axons regenerate and degenerate is critical to our ability to stimulate repair after neuronal injury (46). Here, we identify TIR-1/SARM1/dSarm, a key regulator of axon degeneration, as a previously uncharacterized intrinsic inhibitor of axon regeneration that can be targeted to increase repair of injured motor axons. Our data indicate that to achieve this specificity of function, tir-1 inhibits axon regeneration with the NSY-1 - PMK-1 MAPK pathway on the proximal side of the injury while it promotes degeneration with the DLK-1 - PMK-3 MAPK pathway on the distal side. Together, our findings reveal opposing mechanisms by which TIR-1 regulates the injury response.

\section{TIR-1/SARM1 inhibits axon regeneration independently from its role in axon degeneration}

We found that TIR-1 inhibits axon regeneration and does so independently of its role in degeneration by investigating the injury response in a new model of $C$. elegans motor axon injury. C. elegans has been a powerful axon injury model owing to its genetic tractability, the ability to carry out experiments in vivo with single axon resolution, and its extensive genome conservation with mammals $(30,31,47)$. In addition, the $C$. elegans motor nervous system lacks myelin and glia, enabling investigation of mechanisms that regulate axon regeneration independently of these extrinsic cues. However, C. elegans has not been a robust model of motor axon degeneration because for unknown reasons, when motor axons are cut once, the distal segment remains largely intact in adult animals (32). Instead, we found that severing an axon twice creates a middle fragment that completely degenerates and a proximal segment that is capable of regenerating. 
Hence, the double injury assay combines the tractability of $C$. elegans with the ability to investigate motor axon regeneration and degeneration on either side of the injury. Using this double injury assay, we found that tir-1 both inhibits axon regeneration and promotes degeneration of injured $C$. elegans motor axons.

Either reducing the function of intrinsic inhibitors or changing the amount of extrinsic cues in the environment, such as degeneration and inflammation, can increase an injured axon's ability to regenerate (7-11). Our findings indicate the enhanced regeneration in tir-1 mutants is not a secondary consequence of reduced degeneration. First, axons lacking tir- 1 regenerate more often than wild-type axons after a single injury, even though a single injury does not cause degeneration in either wild-type or tir- 1 mutants. Second, using the double injury assay of motor axon injury in which both axon regeneration and degeneration occur, we found that tir- 1 mutant axons were as likely to regenerate in the presence or absence of degeneration on the other side of the injury. Thus, a decrease in degeneration does not account for the increased regeneration of tir- 1 mutants. Combined with our finding that tir- 1 functions within GABA axons to regulate axon regeneration, our data suggest that tir-1 functions within the proximal stumps of injured GABA axons to regulate axon regeneration.

\section{TIR-1/SARM1 inhibits axon regeneration and degeneration with NSY-1 and DLK-1}

\section{MAPK signaling pathways}

Our finding that tir- 1 regulates both axon regeneration and degeneration raises the question of how this single gene differentially regulates the two seemingly opposite processes in what was once the same axon. Our data indicate that TIR-1/SARM1 carries out these opposing functions by interacting with two divergent MAP kinase signaling pathways on either side of the injury. On the proximal side, TIR-1/SARM1 functions with UNC-43/CAMKII, the NSY-1/ASK1 MAPK 
signaling cascade and the transcription factor DAF-19/RFX to inhibit axon regeneration. The involvement of DAF-19 suggests that TIR-1 activation may change the transcriptional profile of the proximal stump into one that does not support repair. Unlike the TIR-1 pathway that regulates the innate immune response and serotonin biosynthesis, DAF-19 does not require the cotranscription factor ATF-7 to regulate axon regeneration (40). Therefore, TIR-1 likely achieves specificity of function by interacting with particular transcription factors in a temporal and tissue specific manner in response to injury.

On the distal side of the injury, our data suggest TIR-1 regulates degeneration with an alternate MAPK pathway. Loss of $p m k-1$ function has no effect on axon degeneration, nor does it affect the degeneration phenotype of tir-1 loss-of-function mutants, indicating TIR-1 functions independently from the NSY-1 - PMK-1 MAPK pathway to regulate axon degeneration. Instead, the DLK-1 - PMK-3 MAPK pathway is required for the tir- 1 dependent degeneration phenotype in both an injury and a constitutive degeneration model. Similarly, in mice and flies, SARM1 and dSarm interact with the signaling pathways of DLK-1 homologs Wallenda/DLK to regulate axon degeneration, indicating tir-1 likely regulates axon degeneration, at least in part, through evolutionarily conserved mechanisms $(16-18,21)$. To date, how SARM1 ultimately leads to axon degeneration after injury is not completely clear. Besides MAP kinase signaling, effectors of SARM1 function include the downstream Axundead protein and diminished levels of $\mathrm{NAD}^{+}(14$, 15). While there does not appear to be an Axundead homolog in C. elegans, the NADase activity of SARM1 and dSarm has recently been found to be conserved in TIR-1 suggesting TIR-1 may also regulate degeneration through its catalase activity (48).

The finding that TIR-1, a homolog of dSarm and SARM1, essential regulators of axon degeneration, also regulates the opposite response to injury, axon regeneration, is unexpected. In 
addition, TIR-1 was not thought to regulate injury-induced degeneration of C. elegans axons, likely because adult motor axons do not robustly degenerate using the single injury assay and because degeneration of injured mechanosensory axons is regulated by apoptotic engulfment machinery in surrounding tissues, and not by TIR-1 $(32,49)$. The identified dual role of TIR-1 in the injury response raises a number of compelling questions, including: Is TIR-1 differentially activated on either side of the injury to determine whether a fragment repairs itself or selfdestructs? Does TIR-1 regulate regeneration throughout the nervous system, or, like other intrinsic regulators of regeneration, does it function according to cell type and age (50-52)? Finally, can TIR-1 signaling be manipulated to specifically enhance axon regeneration in mammals?

In conclusion, we present a model where TIR-1 functions cell-autonomously in GABA motor neurons to regulate axon regeneration, independently from its role in axon degeneration. We show that to achieve specificity, TIR-1 directs these contrasting functions with separate downstream MAPK signaling pathways on either side of the injury. Understanding how TIR-1 regulates a balance between axon regeneration and degeneration may provide novel ways to push the nervous system towards regeneration in response to injury. 


\section{Materials and Methods}

\section{C. elegans strains and culture}

Unless otherwise noted, all C. elegans strains were maintained at $20^{\circ} \mathrm{C}$ on $\mathrm{NGM}$ plates seeded with OP50 E. coli. Strains listed below were kindly provided by the Alkema, Francis, Hammarlund, Pukkila-Worley labs and the Caenorhabditis Genetics Center, which is funded by NIH Office of Research Infrastructure Programs (P40 OD010440). To visualize GABA motor neurons, strains were crossed into EG1285 (oxIs12 [unc-47p::GFP, lin-15(+)]). Mutants used in this study: tir-1(qd4), tir-1(tm3036), ufIs175(flp-13p::myristoylatedGFP), nsy-1(ag3), pmk1(km25); unc-43(e408), daf-19(m86), dlk-1(ju476), pmk-3(ok169), tol-1(nr2033), atf-7(gk715).

\section{Laser Axotomy, imaging, and quantification}

Axotomy was performed as previously described (49), unless otherwise stated below. Larval stage 4 (L4) worms were mounted on 3\% agarose pads and immobilized with microbeads (Polysciences) or $0.1 \mathrm{mM}$ levamisole. Single injuries were made at the midline of the axon commissure and 1-3 axons were cut per worm. Double injuries were made with two targeted cuts, one roughly halfway between the ventral nerve cord and the midline of the worm and the second roughly halfway between the midline of the worm and the dorsal nerve cord, creating an approximately $25 \mu \mathrm{m}$ middle fragment. The two injuries are performed within seconds of each other in the same order (proximal-distal). One axon was cut per worm in the double injury experiments. Axon regeneration was quantified 24 hours after injury unless otherwise stated by placing animals immobilized with microbeads or $0.1 \mathrm{mM}$ levamisole on a $3 \%$ agarose pad. Animals were imaged with a Nikon 100X 1.4NA objective, Andor Zyla sCMOS camera and Leica EL6000 light source. Regeneration was scored as the percent of axons that regenerated 
fully to the dorsal cord (F), $75 \%$ of the distance to the dorsal cord $\left(\mathrm{M}^{+}\right)$, to the midline $(\mathrm{M})$, to below the midline or had $3+$ filopodia extending from the proximal segment $\left(\mathrm{M}^{-}\right)$. Axons that failed to regenerate, or had 2 or fewer small filopodia protruding from the proximal segment were scored as $\mathrm{N}$ and NF respectively.

\section{CRISPR}

CRISPR gene editing was conducted as previously described (53). crRNA, tracrRNA, and Cas9 were made by IDT and injected with the rol-6 co-injection marker. The TIR1::happylinker::mCherry crRNA sequence used is CTGCATCCACAACTTCCGAT. CRISPR mutants were genotyped and outcrossed before analysis.

\section{TIR-1 expression}

To determine where TIR-1 is expressed we generated transgenic lines expressing TIR-1 under its endogenous promoter by injecting plasmid bamEx102[tir-1p::tir-1b::mCherry::unc-54 3'UTR] and co-injection marker ceh-22::gfp into ABC16 (tir-1(qd4); oxIs 12[unc-47p::GFP, lin-15(+)]). L4 stage animals were imaged on Perkin Elmer Precisely UltraVIEW VoX confocal imaging system.

To determine where TIR-1 functions, we generated transgenic animals expressing TIR-1 specifically in the GABA motor neurons or in the epidermis. GABA specific expression was achieved by injecting plasmid bamEx 100[unc-47p::tir-1b::mCherry::unc-54 3'UTR] with coinjection marker psH21 [str-1p::gfp(+)] into EG1285 (oxIs12[unc-47p::GFP, lin-15(+)]) as previously described (54). Epidermis specific expression was achieved by injecting plasmid bamEx101[dpy-7p::tir-1b::mCherry::unc-54 3'UTR] with co-injection marker psH21[str1p::gfp(+)] into ABC16 (tir-1(qd4); oxIs12[unc-47p::GFP, lin-15(+)]). The resulting transgenic 
strains were crossed into tir-1(qd4) and $d l k-1(j u 476)$ mutants. Axotomies were performed as described above.

\section{Degeneration and TIR-1 localization}

Middle fragment degeneration after double injury was measured 24 hours after injury unless otherwise indicated and grouped into 3 categories: degenerated (complete clearance), partially degenerated ( $>65 \%$ of the middle fragment degenerated) or largely remained intact $(>80 \%$ of middle fragment remained) 24 hours after double injury.

Chronic degeneration and protein localization in the absence of injury was assessed by immobilizing L4 stage worms with $0.1 \mathrm{mM}$ levamisole on a 3\% agarose pad. Images were taken on a Perkin Elmer Precisely UltraVIEW VoX spinning disc confocal imaging system using a 40X objective. Images were compressed and exported as TIFF files for processing in FIJI. Images were rotated and cropped to the same ROI. The axon or dorsal nerve cord segment to be analyzed was traced using a segmented line ROI and mean intensity was quantified.

\section{Statistical Analysis}

Statistical analysis was performed using GraphPad QuickCalcs (www.graphpad.com/quickcals/) and Prism (GraphPad). Categorical data: data from repeated assays comparing a mutant and corresponding control strain were pooled for statistical analysis. Bars represent $95 \%$ confidence intervals and significance determined with Fisher's exact test, where $* \mathrm{p}<0.05, * * \mathrm{p}<0.01, * * * \mathrm{p}$ $<0.001$. Continuous data (fluorescence intensity of GFP along axon commissure) are presented as the mean with error bars representing the standard error of the mean. Significance was calculated with T-tests, where $* \mathrm{p}<0.05, * * \mathrm{p}<0.01, * * * \mathrm{p}<0.001$. 


\section{Acknowledgments:}

Author contributions: V. Julian contributed to experimental design, investigation, interpretation, and manuscript preparation. A. Byrne contributed to experimental design, interpretation, and manuscript preparation. We thank A. Zeamer for technical contribution to Figure S2, W. Joyce and M. Liu for construction of reagents, along with M. Alkema and V. Budnik for helpful comments on the manuscript. Competing interests: Authors have no competing interests. Data and materials availability: All data is available in the main text or the supplementary materials. All materials used in the analysis are available upon request. 


\section{References}

1. S. David, A. J. Aguayo, Axonal elongation into peripheral nervous system "bridges" after central nervous system injury in adult rats. Science 214, 931-933 (1981).

2. D. Cai et al., Neuronal cyclic AMP controls the developmental loss in ability of axons to regenerate. J Neurosci 21, 4731-4739 (2001).

3. A. Kaplan, S. Ong Tone, A. E. Fournier, Extrinsic and intrinsic regulation of axon regeneration at a crossroads. Front Mol Neurosci 8, 27 (2015).

4. M. Mahar, V. Cavalli, Intrinsic mechanisms of neuronal axon regeneration. Nat Rev Neurosci 19, 323-337 (2018).

5. T. C. Sutherland, C. G. Geoffroy, The Influence of Neuron-Extrinsic Factors and Aging on Injury Progression and Axonal Repair in the Central Nervous System. Front Cell Dev Biol 8, 190 (2020).

6. J. Silver, M. E. Schwab, P. G. Popovich, Central nervous system regenerative failure: role of oligodendrocytes, astrocytes, and microglia. Cold Spring Harb Perspect Biol 7, a020602 (2014).

7. M. E. Schwab, S. M. Strittmatter, Nogo limits neural plasticity and recovery from injury. Curr Opin Neurobiol 27, 53-60 (2014).

8. Z. He, Y. Jin, Intrinsic Control of Axon Regeneration. Neuron 90, 437-451 (2016).

9. M. Curcio, F. Bradke, Axon Regeneration in the Central Nervous System: Facing the Challenges from the Inside. Annu Rev Cell Dev Biol 34, 495-521 (2018).

10. A. P. Tran, P. M. Warren, J. Silver, The Biology of Regeneration Failure and Success After Spinal Cord Injury. Physiol Rev 98, 881-917 (2018). 
11. A. Tedeschi, F. Bradke, Spatial and temporal arrangement of neuronal intrinsic and extrinsic mechanisms controlling axon regeneration. Curr Opin Neurobiol 42, 118-127 (2017).

12. J. M. Osterloh et al., dSarm/Sarm1 is required for activation of an injury-induced axon death pathway. Science 337, 481-484 (2012).

13. J. Gerdts, D. W. Summers, Y. Sasaki, A. DiAntonio, J. Milbrandt, Sarm1-mediated axon degeneration requires both SAM and TIR interactions. J Neurosci 33, 13569-13580 (2013).

14. L. J. Neukomm et al., Axon Death Pathways Converge on Axundead to Promote Functional and Structural Axon Disassembly. Neuron 95, 78-91 e75 (2017).

15. K. Essuman et al., The SARM1 Toll/Interleukin-1 Receptor Domain Possesses Intrinsic $\mathrm{NAD}(+)$ Cleavage Activity that Promotes Pathological Axonal Degeneration. Neuron 93, 1334-1343 e1335 (2017).

16. J. Yang et al., Pathological axonal death through a MAPK cascade that triggers a local energy deficit. Cell 160, 161-176 (2015).

17. L. J. Walker et al., MAPK signaling promotes axonal degeneration by speeding the turnover of the axonal maintenance factor NMNAT2. Elife 6, (2017).

18. J. Gerdts, D. W. Summers, J. Milbrandt, A. DiAntonio, Axon Self-Destruction: New Links among SARM1, MAPKs, and NAD+ Metabolism. Neuron 89, 449-460 (2016).

19. J. Gerdts, E. J. Brace, Y. Sasaki, A. DiAntonio, J. Milbrandt, SARM1 activation triggers axon degeneration locally via NAD(+) destruction. Science 348, 453-457 (2015).

20. J. E. Shin et al., SCG10 is a JNK target in the axonal degeneration pathway. Proc Natl Acad Sci U S A 109, E3696-3705 (2012). 
21. D. W. Summers, E. Frey, L. J. Walker, J. Milbrandt, A. DiAntonio, DLK Activation Synergizes with Mitochondrial Dysfunction to Downregulate Axon Survival Factors and Promote SARM1-Dependent Axon Degeneration. Mol Neurobiol 57, 1146-1158 (2020).

22. S. Geisler et al., Gene therapy targeting SARM1 blocks pathological axon degeneration in mice. J Exp Med 216, 294-303 (2019).

23. M. A. White et al., Sarm1 deletion suppresses TDP-43-linked motor neuron degeneration and cortical spine loss. Acta Neuropathol Commun 7, 166 (2019).

24. J. Veriepe, L. Fossouo, J. A. Parker, Neurodegeneration in C. elegans models of ALS requires TIR-1/Sarm1 immune pathway activation in neurons. Nat Commun 6, 7319 (2015).

25. E. Lezi et al., An Antimicrobial Peptide and Its Neuronal Receptor Regulate Dendrite Degeneration in Aging and Infection. Neuron 97, 125-138 e125 (2018).

26. N. Pujol et al., Distinct innate immune responses to infection and wounding in the C. elegans epidermis. Curr Biol 18, 481-489 (2008).

27. R. P. Shivers, T. Kooistra, S. W. Chu, D. J. Pagano, D. H. Kim, Tissue-specific activities of an immune signaling module regulate physiological responses to pathogenic and nutritional bacteria in C. elegans. Cell Host Microbe 6, 321-330 (2009).

28. C. F. Chuang, C. I. Bargmann, A Toll-interleukin 1 repeat protein at the synapse specifies asymmetric odorant receptor expression via ASK1 MAPKKK signaling. Genes Dev 19, 270$281(2005)$

29. N. T. Liberati et al., Requirement for a conserved Toll/interleukin-1 resistance domain protein in the Caenorhabditis elegans immune response. Proc Natl Acad Sci U S A 101, 6593-6598 (2004). 
30. A. B. Byrne, M. Hammarlund, Axon regeneration in C. elegans: Worming our way to mechanisms of axon regeneration. Exp Neurol 287, 300-309 (2017).

31. M. F. Yanik et al., Neurosurgery: functional regeneration after laser axotomy. Nature 432, 822 (2004).

32. A. L. A. Nichols et al., The Apoptotic Engulfment Machinery Regulates Axonal Degeneration in C. elegans Neurons. Cell Rep 14, 1673-1683 (2016).

33. C. Couillault et al., TLR-independent control of innate immunity in Caenorhabditis elegans by the TIR domain adaptor protein TIR-1, an ortholog of human SARM. Nat Immunol 5, 488-494 (2004).

34. M. Hammarlund, P. Nix, L. Hauth, E. M. Jorgensen, M. Bastiani, Axon regeneration requires a conserved MAP kinase pathway. Science 323, 802-806 (2009).

35. D. Yan, Z. Wu, A. D. Chisholm, Y. Jin, The DLK-1 kinase promotes mRNA stability and local translation in C. elegans synapses and axon regeneration. Cell 138, 1005-1018 (2009).

36. A. Sagasti et al., The CaMKII UNC-43 activates the MAPKKK NSY-1 to execute a lateral signaling decision required for asymmetric olfactory neuron fates. Cell 105, 221-232 (2001).

37. A. E. Ghiretti et al., Rem2 is an activity-dependent negative regulator of dendritic complexity in vivo. J Neurosci 34, 392-407 (2014).

38. F. Xi et al., Calcium/calmodulin-dependent protein kinase II regulates mammalian axon growth by affecting F-actin length in growth cone. J Cell Physiol 234, 23053-23065 (2019).

39. S. H. Chung et al., Novel DLK-independent neuronal regeneration in Caenorhabditis elegans shares links with activity-dependent ectopic outgrowth. Proc Natl Acad Sci US A 113, E2852-2860 (2016). 
40. Y. Xie, M. Moussaif, S. Choi, L. Xu, J. Y. Sze, RFX transcription factor DAF-19 regulates 5-HT and innate immune responses to pathogenic bacteria in Caenorhabditis elegans. PLoS Genet 9, e1003324 (2013).

41. A. Ghosh-Roy, Z. Wu, A. Goncharov, Y. Jin, A. D. Chisholm, Calcium and cyclic AMP promote axonal regeneration in Caenorhabditis elegans and require DLK-1 kinase. $J$ Neurosci 30, 3175-3183 (2010).

42. B. R. Miller et al., A dual leucine kinase-dependent axon self-destruction program promotes Wallerian degeneration. Nat Neurosci 12, 387-389 (2009).

43. X. Xiong et al., The Highwire ubiquitin ligase promotes axonal degeneration by tuning levels of Nmnat protein. PLoS Biol 10, e1001440 (2012).

44. X. Xiong et al., Protein turnover of the Wallenda/DLK kinase regulates a retrograde response to axonal injury. J Cell Biol 191, 211-223 (2010).

45. D. S. Welsbie et al., Functional genomic screening identifies dual leucine zipper kinase as a key mediator of retinal ganglion cell death. Proc Natl Acad Sci U S A 110, 4045-4050 (2013).

46. M.P. Girouard et al., The Molecular Interplay between Axon Degeneration and Regeneration. Developmental Neurobiology 78, 978-990 (2018).

47. W. Kim, R. S. Underwood, I. Greenwald, D. D. Shaye, OrthoList 2: A New Comparative Genomic Analysis of Human and Caenorhabditis elegans Genes. Genetics 210, 445-461 (2018).

48. H. S. Loring, J. D. Icso, V. V. Nemmara, P. R. Thompson, Initial Kinetic Characterization of Sterile Alpha and Toll/Interleukin Receptor Motif-Containing Protein 1. Biochemistry 59, 933-942 (2020). 
49. A. B. Byrne, T. J. Edwards, M. Hammarlund, In vivo laser axotomy in C. elegans. J Vis Exp, (2011).

50. A. B. Byrne et al., Insulin/IGF1 signaling inhibits age-dependent axon regeneration. Neuron $81,561-573$ (2014).

51. C. G. Geoffroy, B. J. Hilton, W. Tetzlaff, B. Zheng, Evidence for an Age-Dependent Decline in Axon Regeneration in the Adult Mammalian Central Nervous System. Cell Rep 15, 238246 (2016).

52. X. Duan et al., Subtype-specific regeneration of retinal ganglion cells following axotomy: effects of osteopontin and mTOR signaling. Neuron 85, 1244-1256 (2015).

53. G. A. Dokshin, K. S. Ghanta, K. M. Piscopo, C. C. Mello, Robust Genome Editing with Short Single-Stranded and Long, Partially Single-Stranded DNA Donors in Caenorhabditis elegans. Genetics 210, 781-787 (2018).

54. C. C. Mello, J. M. Kramer, D. Stinchcomb, V. Ambros, Efficient gene transfer in C.elegans: extrachromosomal maintenance and integration of transforming sequences. EMBO J10, 3959-3970 (1991). 
A

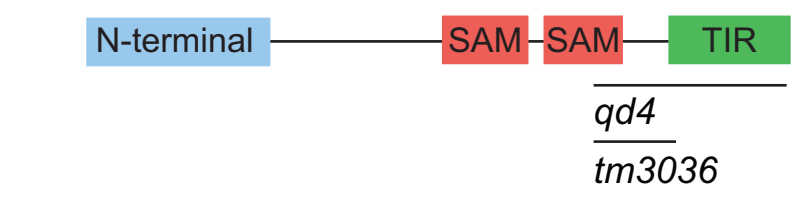

\section{C}
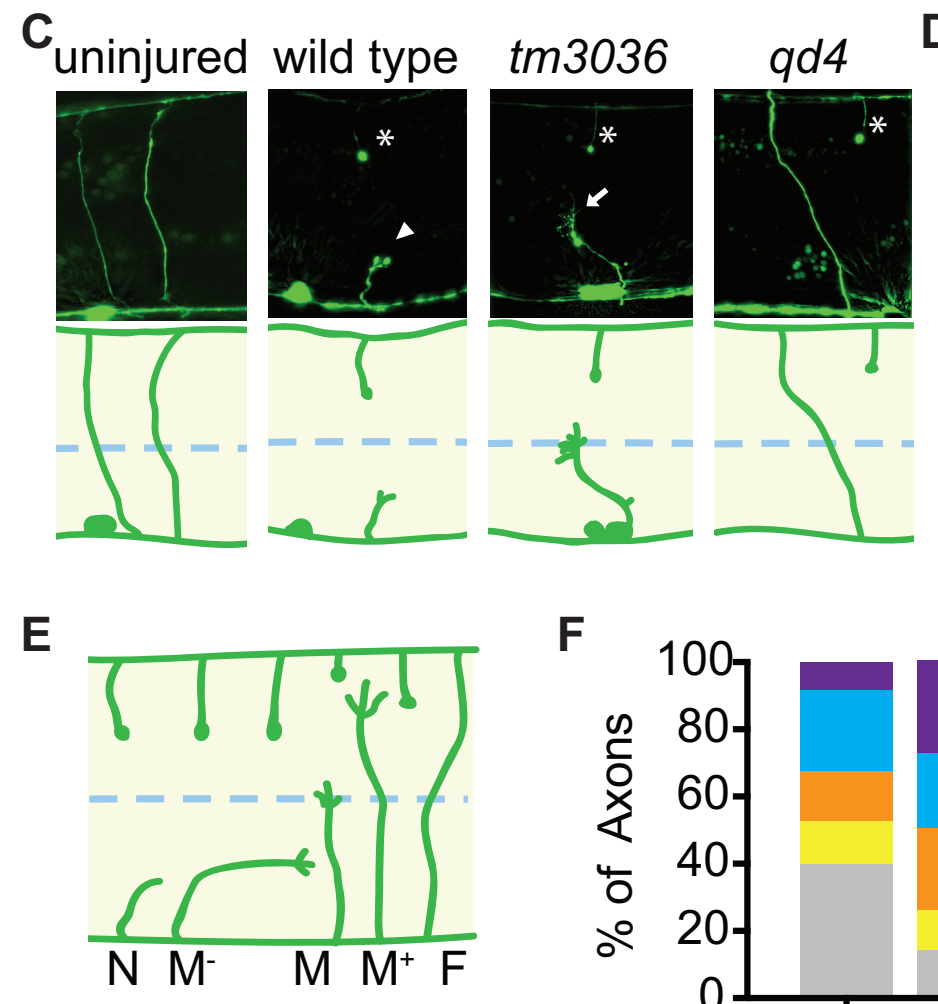

$\mathbf{F}$
B

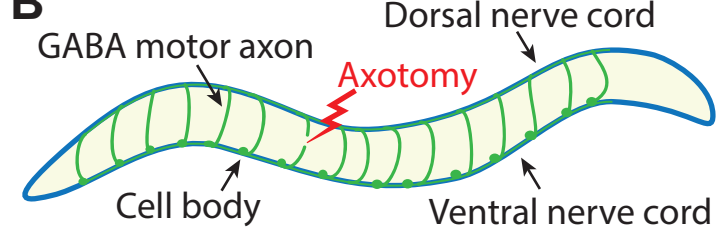

D
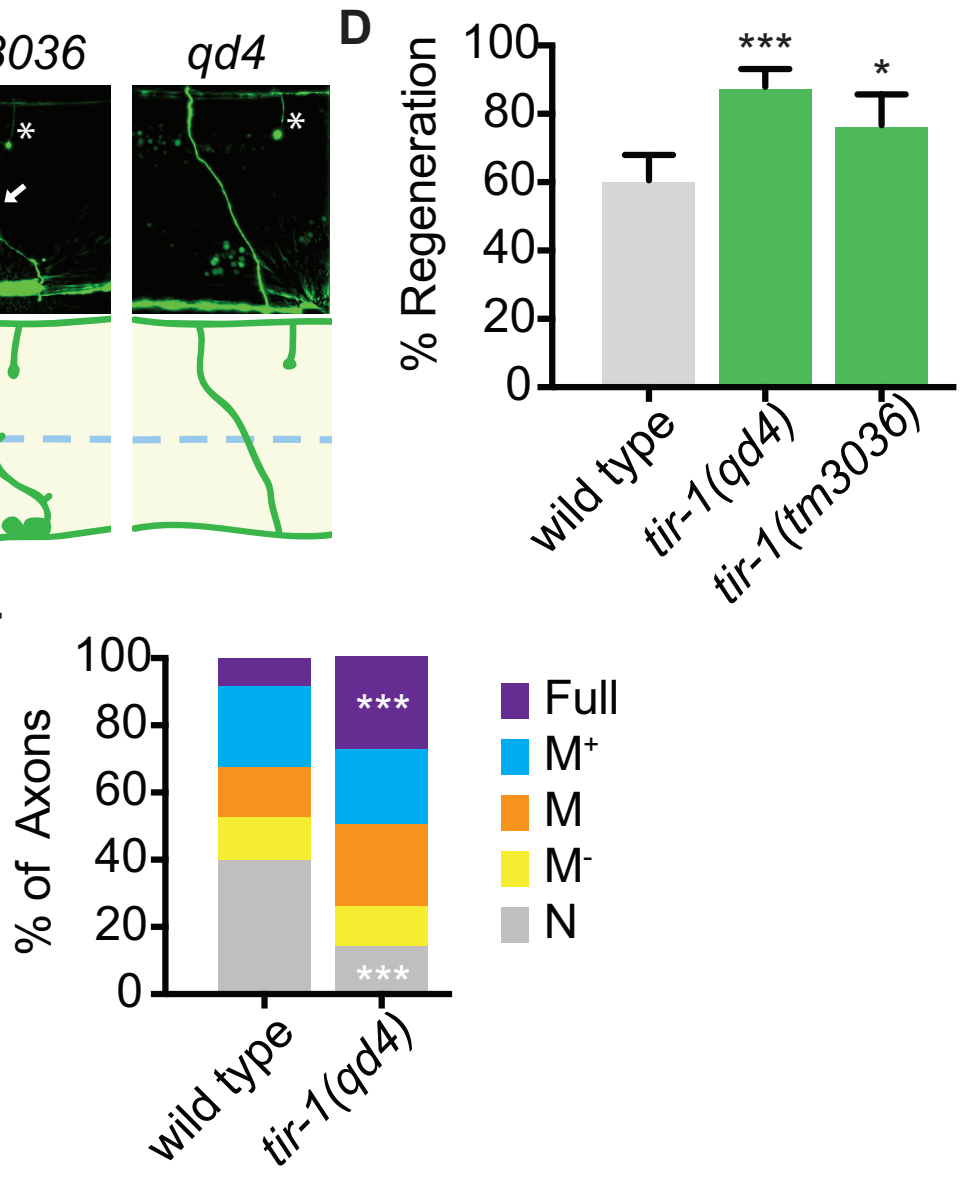

Figure 1. tir-1 inhibits axon regeneration. (A) TIR-1 contains an N-terminal auto-inhibitory domain, two sterile alpha motif (SAM) domains and a toll-interleukin-1 receptor (TIR) domain. Null alleles $q d 4$ and tm3036 disrupt the essential TIR domain. (B) C. elegans GABA motor neurons are axotomized to study the injury response in vivo and with single axon resolution. (C) Representative GABA motor neurons before and 24 hours after single laser injury at the lateral midline of the animal (dashed line). Asterisks indicate distal stumps, arrowheads indicate retraction bulbs, and arrows indicate growth cones. (D) More axons regenerate in tir-1 mutants 
compared to wild-type animals, as measured by the percentage of axons that form a growth cone 24 hours after laser surgery. (E) Regeneration phenotypes were categorized according to whether regenerating axons reached landmarks $\mathrm{M}^{-}, \mathrm{M}, \mathrm{M}^{+}, \mathrm{F}$, or failed to form a growth cone, N. (F) More tir-1(-) axons initiate regeneration and reach the dorsal cord compared to wild-type axons, as indicated by a significant reduction in the number of axons that failed to form a growth cone (N) and a significant increase in the number of fully regenerated axons (Full). Significance relative to wild type is indicated by $* \mathrm{p}<0.05, * * * \mathrm{p}<0.001$, Fisher's exact test. Error bars represent $95 \%$ confidence intervals. 


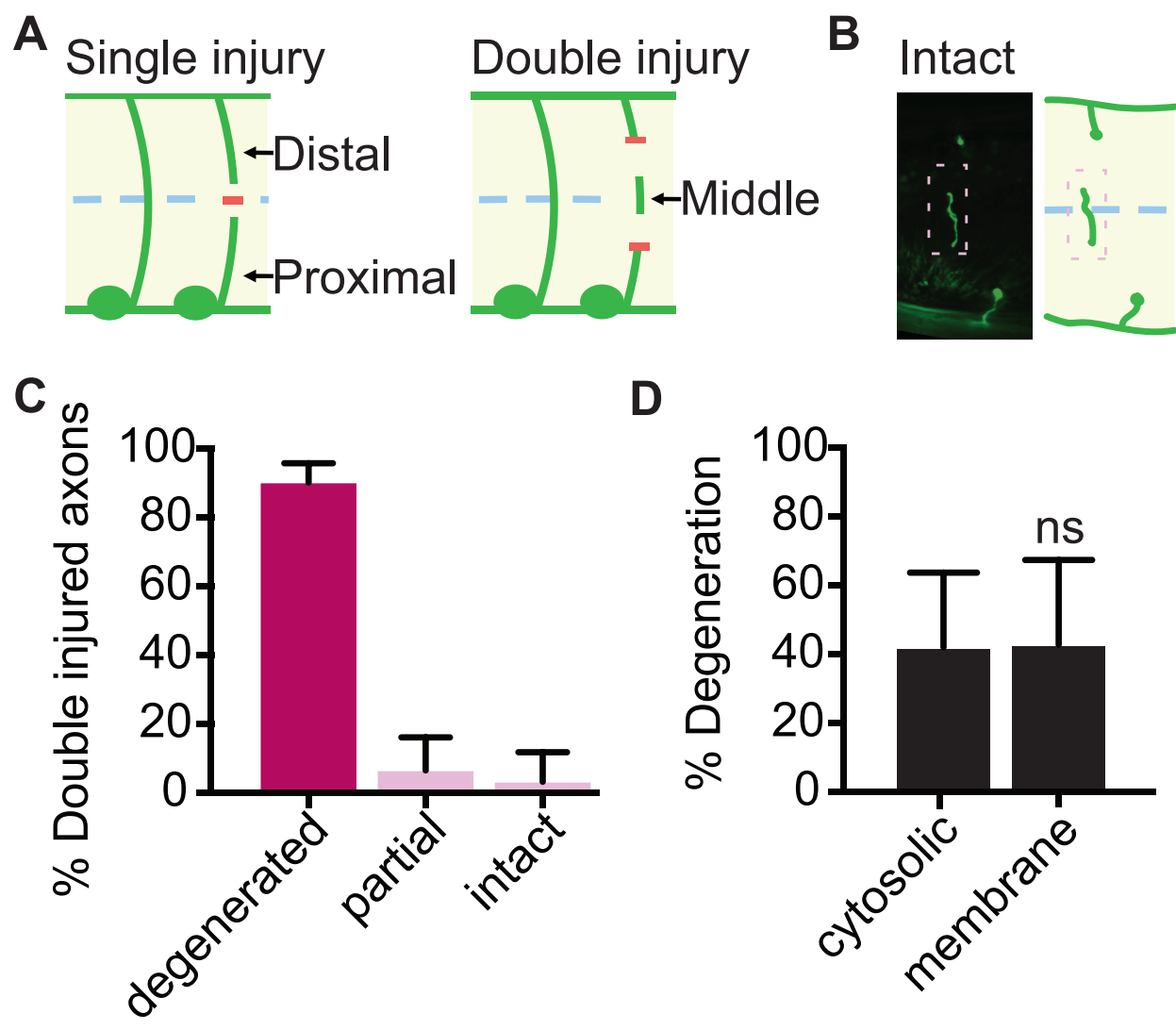

Figure 2. Double injury induces axon degeneration in GABA motor neurons. (A) Single and double injury models. After being severed once, axons do not degenerate. When severed twice, the middle segment degenerates. (B) Micrographs of intact and degenerated middle fragments 24 hours post axotomy. Boxes with dashed lines highlight intact (pink) and degenerated (gray) middle fragments. (C) Most middle fragments degenerate 24 hours after double injury. (D) No significant difference in middle fragment degeneration was observed between axons expressing membrane tagged- or cytosolic GFP 1.5 hours after injury, which represents an active time of degeneration at which approximately half of the severed axons are no longer visible. ns $=$ no significance. Error bars represent $95 \%$ confidence intervals. 
A

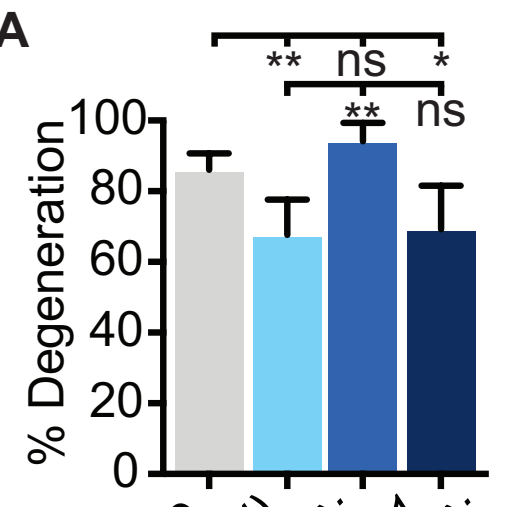

$E$

E)

inil
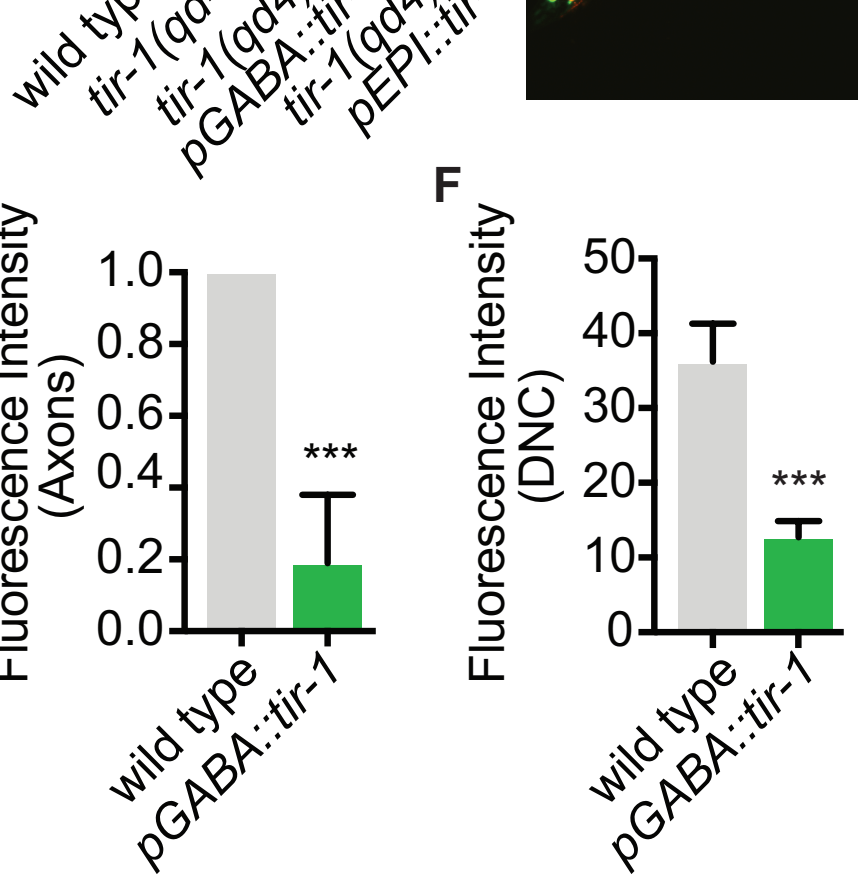

$\mathrm{H}$

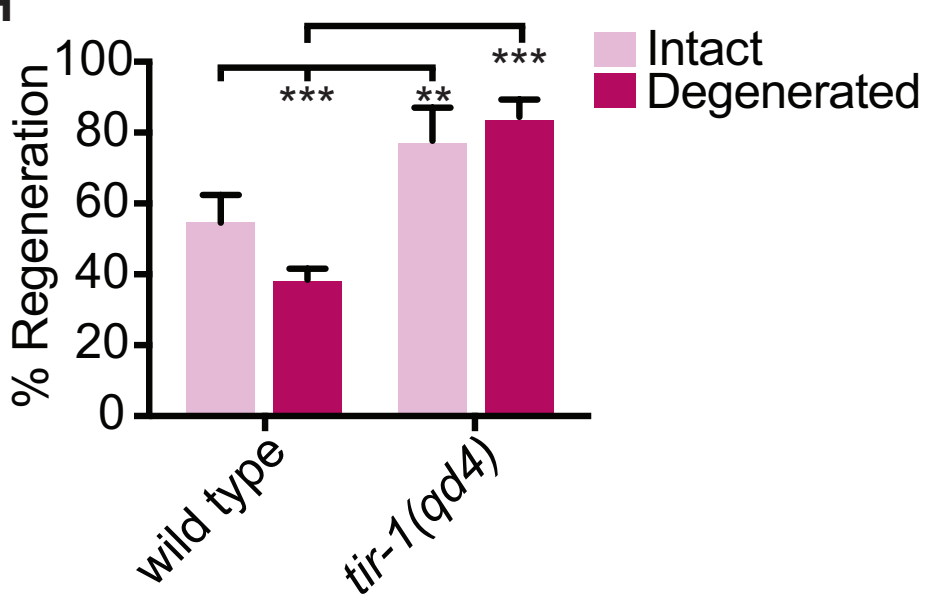

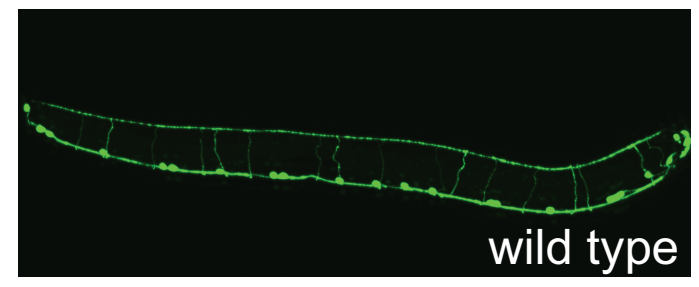

C
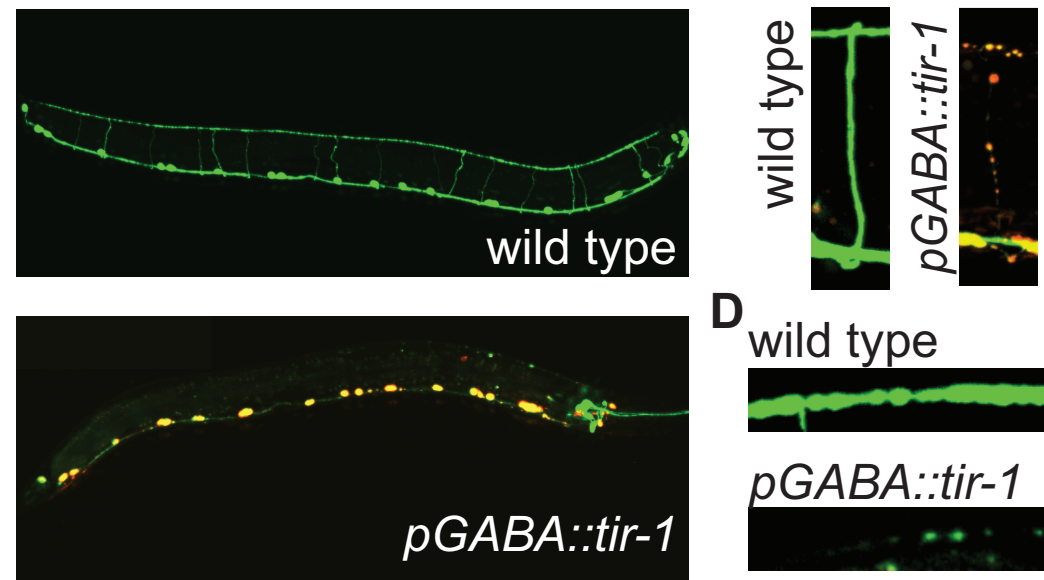

wild type

G

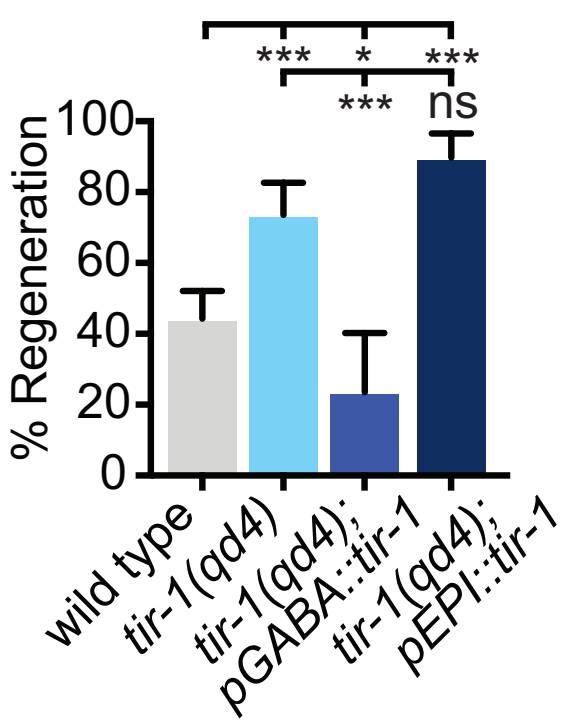

Figure 3. tir-1 functions cell-autonomously to regulate the injury response. (A) GABA specific expression of tir- $1 b$ rescues axon degeneration in tir- $1(q d 4)$ mutants. (B) GABA axons seen in 
whole worm micrographs of wild-type animals (above) appear degenerated in transgenic animals expressing unc-47p::tir-1::mCherry. (C) Individual axons and (D) dorsal nerve cords of wild-type and transgenic animals expressing unc-47p::tir-1::mCherry. (E) Normalized GFP intensity along axon commissures and (F) GFP intensity along the dorsal nerve cord is decreased in animals that overexpress tir-1 in GABA neurons, indicating tir-1 promotes neuronal degeneration. (F) GABA specific expression of $t i r-1 b$ rescues axon regeneration in tir- $1(q d 4)$ mutants. (G) Regeneration is inhibited when the middle fragment degenerates in wild-type animals, but not in tir-1(qd4) mutants. Significance relative to wild type or $\operatorname{tir}-1(q d 4)$ is indicated by $* p<0.05, * * p<0.01$, $* * * p<0.001$. Error bars represent $95 \%$ confidence intervals. 


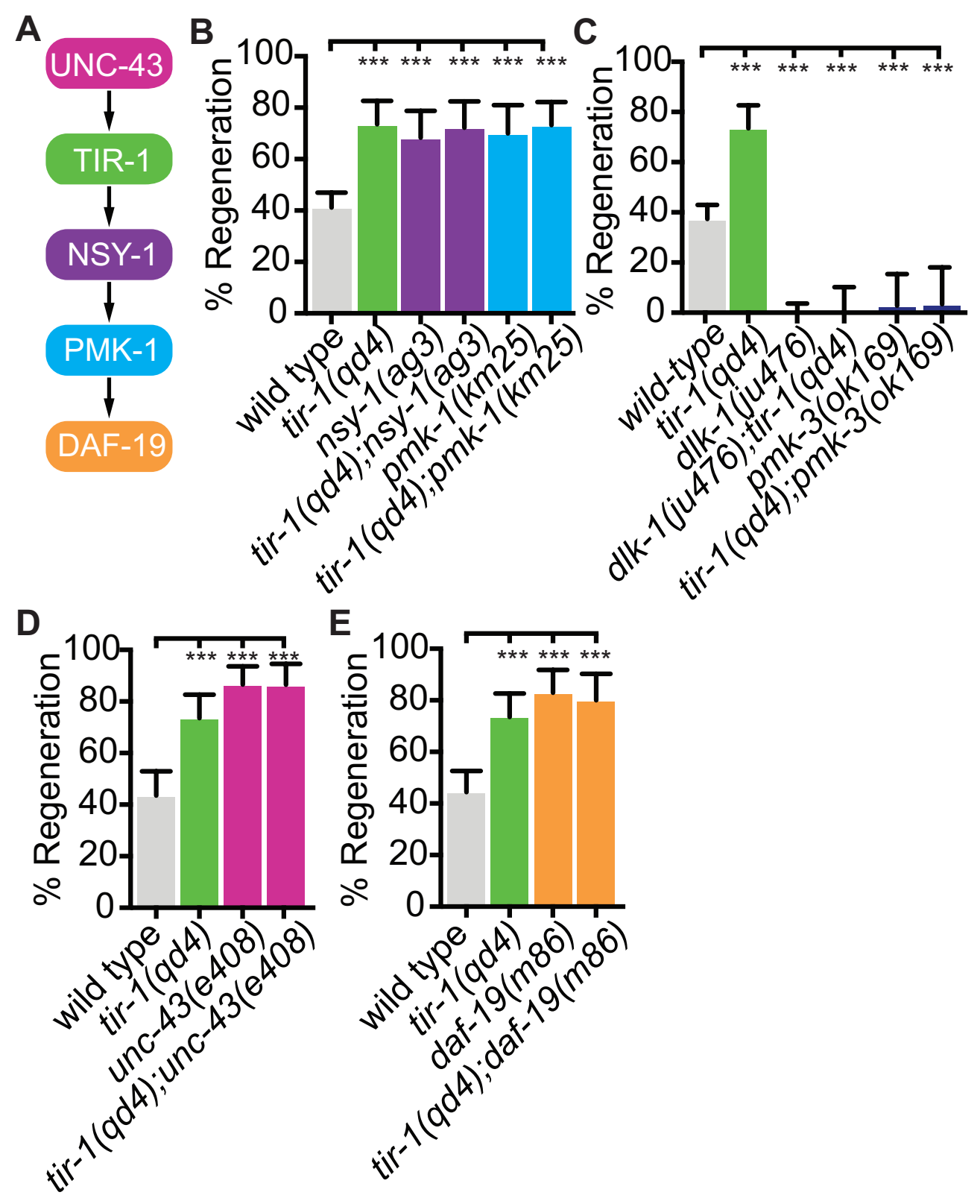

Figure 4. tir-1 functions with the $n s y-1$ signaling pathway to inhibit axon regeneration. (A) Components of the TIR-1- NSY-1 signaling cascade. (B) Axon regeneration is significantly increased in predicted null alleles of $n s y-1 / A S K 1$ and $p m k-1 / p 38$ following double injury, in both the presence and absence of tir-1 function. Double mutants are not statistically different from single mutants. Null mutation of unc-43/CAMIIK enhances axon regeneration after double injury in the presence and absence of the tir-1(qd4) mutation. (D) Loss of the transcription factor $d a f$ - 
bioRxiv preprint doi: https://doi.org/10.1101/2020.06.23.165852; this version posted June 23, 2020. The copyright holder for this preprint (which was not certified by peer review) is the author/funder. All rights reserved. No reuse allowed without permission.

19/RFX1-3 increases axon regeneration after double injury in the presence and absence of tir-1.

Significance relative to wild type is indicated by $* \mathrm{p}<0.05, * * \mathrm{p}<0.01, * * * \mathrm{p}<0.001$. Error bars represent $95 \%$ confidence intervals. 
A

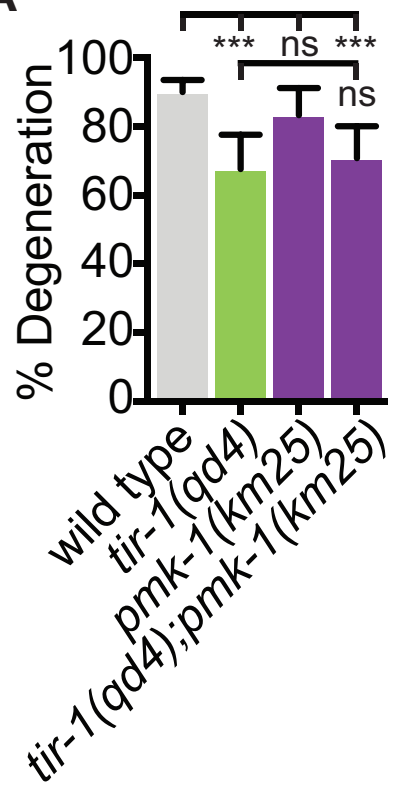

B

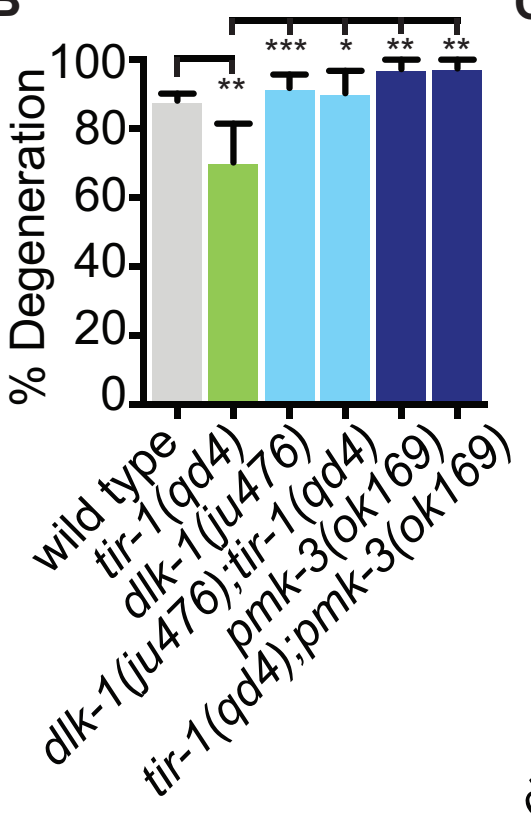

C

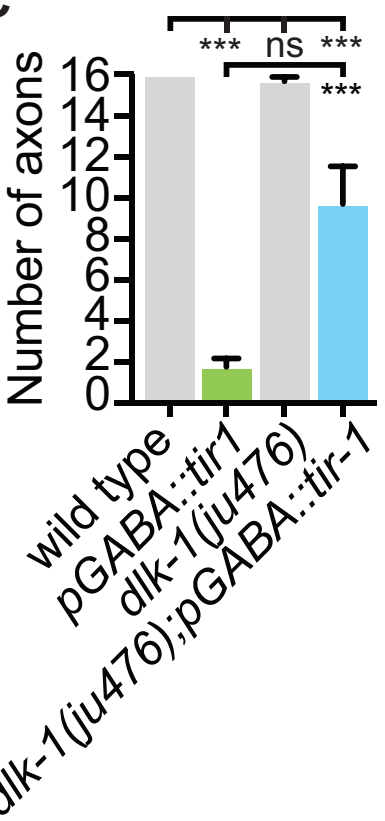

D

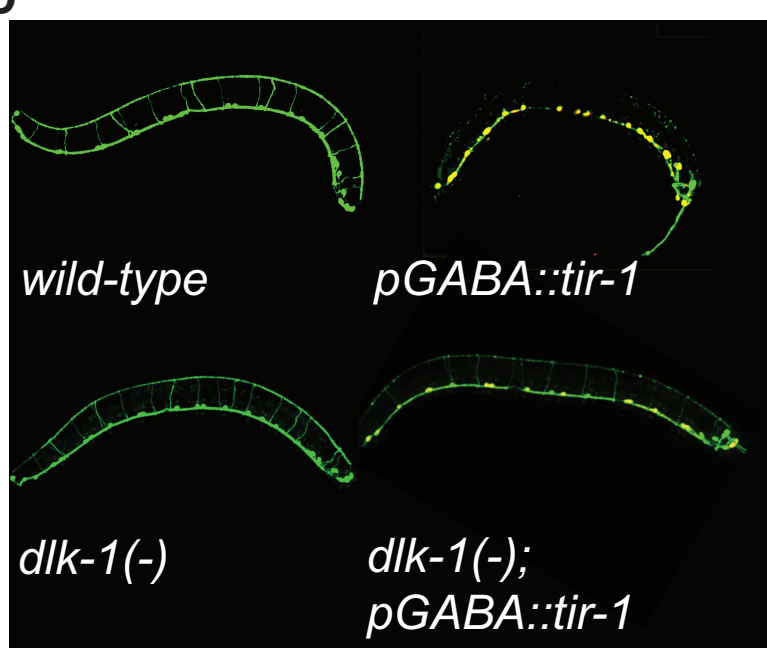

E

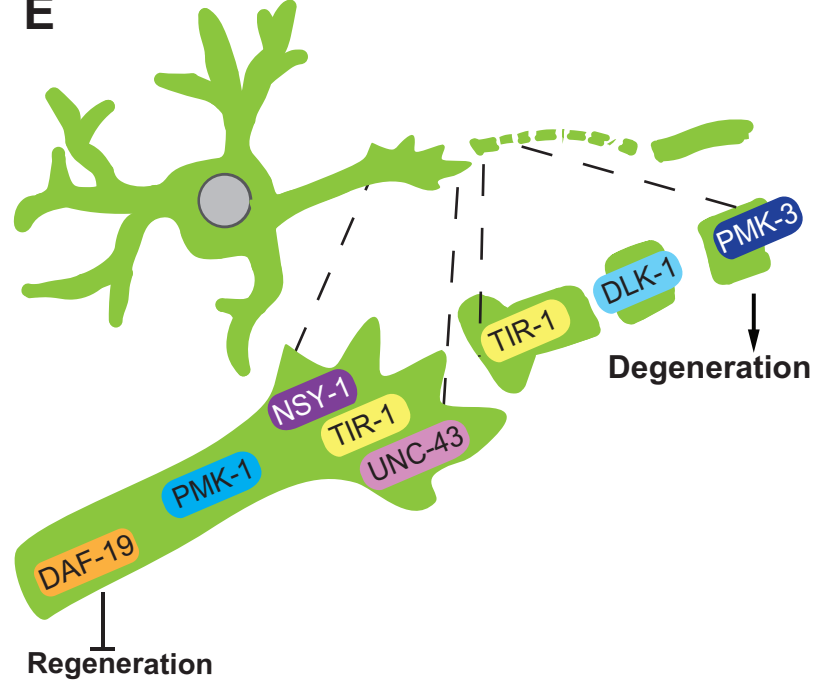

Figure 5. tir-1 functions with the $d l k-1$ signaling pathway to regulate axon degeneration. (A)

Loss of $p m k-1 / p 38$ does not affect degeneration with or without tir-1 function. (B) $d l k-1 / D L K / L Z K$ MAP3K and the downstream pmk-3 p38 MAPK are required for the degeneration phenotype of tir-1 mutants. (C, D) Loss of $d l k-1$ suppresses the increased degeneration caused by overexpressing tir-1 in uninjured GABA neurons. (E) TIR-1 inhibits axon regeneration and axon degeneration in the same injured axon. In the proximal stump, TIR-1 functions with the NSY-1 signaling cascade 
bioRxiv preprint doi: https://doi.org/10.1101/2020.06.23.165852; this version posted June 23, 2020. The copyright holder for this preprint (which was not certified by peer review) is the author/funder. All rights reserved. No reuse allowed without permission.

to modify gene transcription and inhibit axon regeneration. In the severed fragment, TIR-1 functions with the DLK-1 signaling cascade to promote axon degeneration. Significance relative to wild type or tir-1(qd4) is indicated by $* \mathrm{p}<0.05, * * \mathrm{p}<0.01, * * * \mathrm{p}<0.001$. Error bars represent $95 \%$ confidence intervals. 


\section{Supplementary Figures}

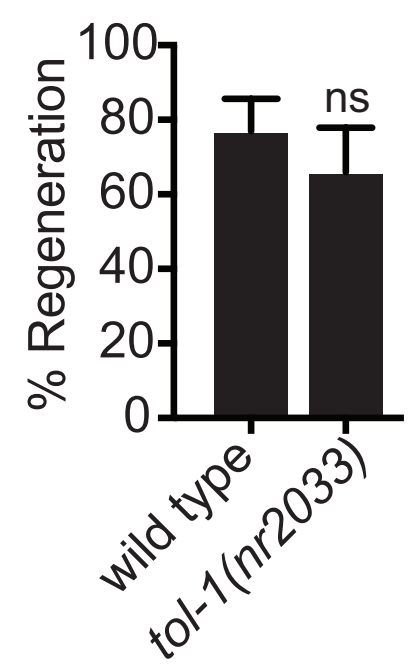

Supplementary Figure 1. tol-1 does not regulate axon regeneration. (A) Axon regeneration is not significantly different in tol-1(nr2033) mutants compared to wild-type controls 24 hours after laser surgery. Error bars represent 95\% confidence intervals. 


\section{A CRISPR tag}
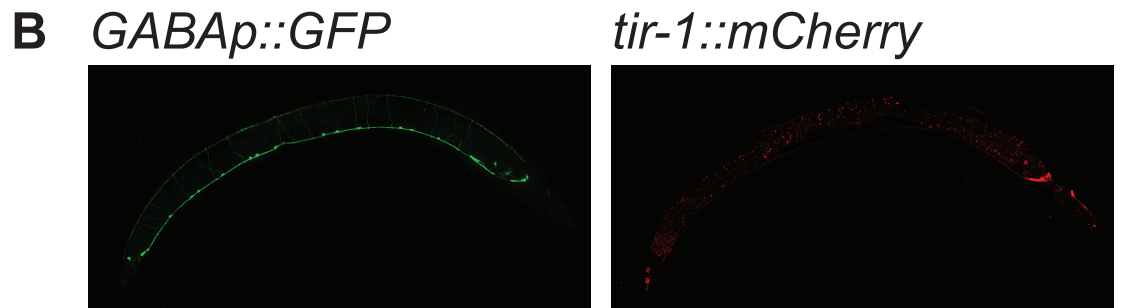

merged

C GABAp::GFP

tir-1::mCherry
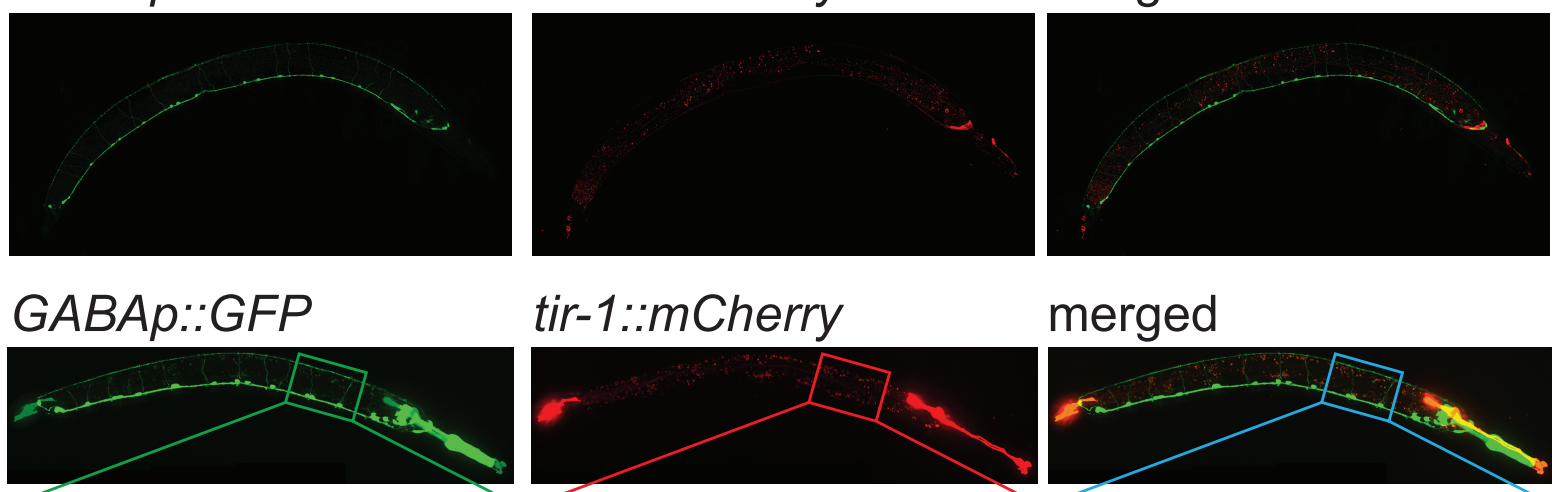

merged
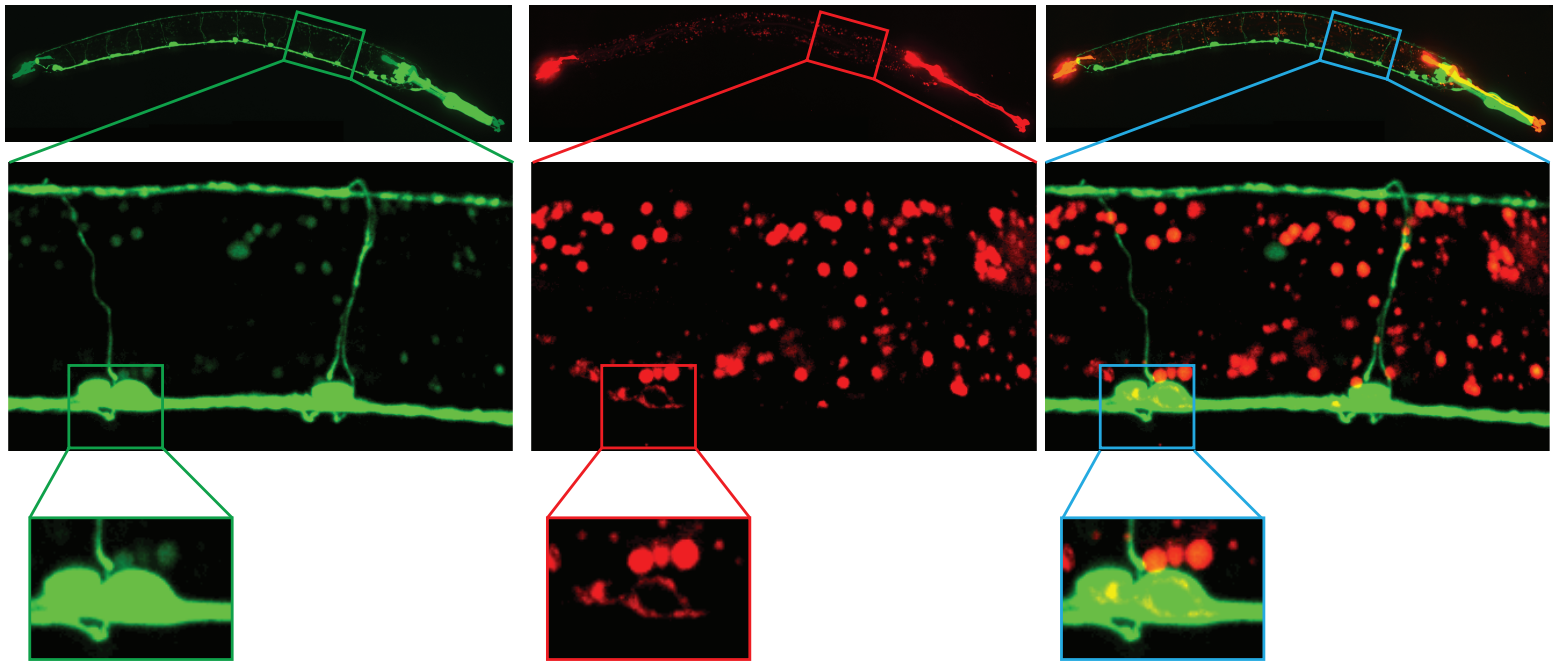

Supplementary Figure 2. TIR-1 localizes to GABA motor neurons. (A) The tir-1 gene was

CRISPR tagged with mCherry. (B) Representative whole worm micrographs of animals expressing endogenous tir-1::mCherry. No overlap was seen with the GABA specific GFP marker $u n c-47 p:: G F P$. (C) Representative micrographs of animals expressing a tir-1b::mCherry extrachromosomal array and the GABA specific GFP marker unc-47p::GFP. TIR-1b::mCherry was observed in cell bodies of GABA neurons (enlarged boxes). 


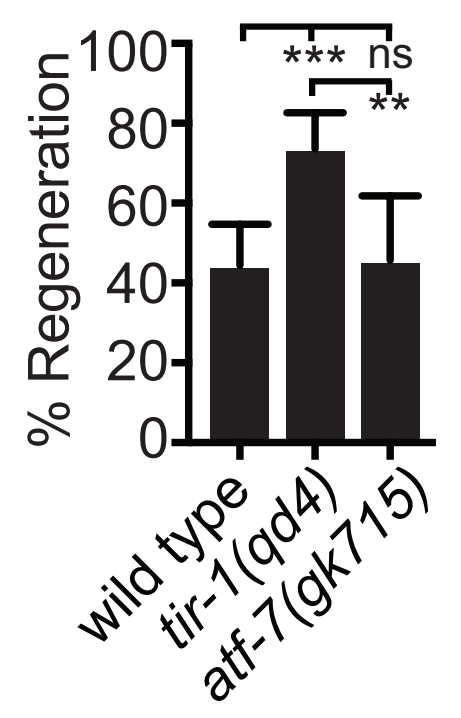

Supplementary Figure 3. The atf-7 transcription factor does not regulate axon regeneration.

(A) Axon regeneration is not significantly different between atf-7(gk715) mutants and wild-type controls 24 hours after laser surgery. Significance relative to wild type or tir-1(qd4) is indicated by $* * \mathrm{p}<0.01, * * * \mathrm{p}<0.001$. Error bars represent $95 \%$ confidence intervals. 